\title{
Practical approach to the patient with hypereosinophilia
}

\section{Citation}

Roufosse, Florence, and Peter F. Weller. 2010. Practical Approach to the Patient with Hypereosinophilia. Journal of Allergy and Clinical Immunology 126, no. 1: 39-44. doi:10.1016/ j.jaci.2010.04.011.

\section{Published Version}

doi:10.1016/j.jaci.2010.04.011

\section{Permanent link}

http://nrs.harvard.edu/urn-3:HUL.InstRepos:33730190

\section{Terms of Use}

This article was downloaded from Harvard University's DASH repository, and is made available under the terms and conditions applicable to Other Posted Material, as set forth at http:// nrs.harvard.edu/urn-3:HUL.InstRepos:dash.current.terms-of-use\#LAA

\section{Share Your Story}

The Harvard community has made this article openly available.

Please share how this access benefits you. Submit a story.

Accessibility 


\title{
Practical approach to the patient with hypereosinophilia
}

\author{
Florence Roufosse, MD $^{\mathrm{a}}$ and Peter F. Weller, M.D. ${ }^{\mathrm{b}}$ \\ a Department of Internal Medicine, Erasme Hospital, Université Libre de Bruxelles, Brussels, \\ Belgium, and Institute for Medical Immunology, Université Libre de Bruxelles, Gosselies, Belgium \\ b Department of Medicine, Division of Infectious Diseases and Division of Allergy and Inflammation, \\ Harvard Medical School, Beth Israel Deaconess Medical Center, Boston Mass. USA
}

\begin{abstract}
Markedly increased blood eosinophilia, $\geq 1.5 \times 10^{9} / \mathrm{L}$, whether discovered fortuitously or found with signs and symptoms of associated organ involvement, commands diagnostic evaluation and often therapeutic interventions. This degree of hypereosinophilia is often, but not uniformly, associated with eosinophilic infiltration of tissues that can potentially lead to irreversible, life-threatening organ damage. Initial approaches focus on ascertaining that eosinophilia is not secondary to other underlying disease processes, including helminth parasite infections, varied types of adverse reactions to medications, and other eosinophil-associated syndromes, such as eosinophilic gastroenteritides, eosinophilic pneumonias and Churg-Strauss syndrome vasculitis. If evaluations exclude eosinophilia attributable to secondary etiologies or other eosinophil-related syndromes or organ-specific diseases, attention must be directed to considerations of varied other forms of the hypereosinophilic syndromes, that include myeloproliferative variants, lymphocytic variants and many of still unknown etiologies. Cognizant of the capacities of eosinophils to mediate tissue damage, the varied etiologies for hypereosinophilia are considered and a contemporary step-wise practical approach to the diagnosis and treatment of patients with hypereosinophilia is presented.
\end{abstract}

\section{Keywords}

eosinophils; hypereosinophilic syndromes; FIPIL1-PDGFRA

\section{Introduction}

Eosinophilia is not an uncommon finding in clinical practice and, when found in association with a constellation of signs and symptoms, can serve as a very useful clue for differential diagnosis. Varied disorders and etiologies may underlie increases in blood eosinophilia. Common causes of eosinophilia include helminthic parasite infections, atopic and allergic diseases and adverse drug reactions. Additional etiologies for more pronounced eosinophilia in variant hypereosinophilic syndromes (HES) merit consideration based on recent newer diagnostic and therapeutic considerations. The defining criteria and sub-classifications of hypereosinophilic syndromes are currently being revised on the basis of modern diagnostic

(C) 2010 American Academy of Allergy, Asthma and Immunology. Published by Mosby, Inc. All rights reserved. Corresponding author: Peter F. Weller, MD Beth Israel Deaconess Medical Center, CLS 943330 Brookline Avenue Boston, MA 02215 Tel: 617-735-4110 FAX: 617-735-4115 pweller@bidmc.harvard.edu.

Publisher's Disclaimer: This is a PDF file of an unedited manuscript that has been accepted for publication. As a service to our customers we are providing this early version of the manuscript. The manuscript will undergo copyediting, typesetting, and review of the resulting proof before it is published in its final citable form. Please note that during the production process errors may be discovered which could affect the content, and all legal disclaimers that apply to the journal pertain. 
tools and advances in pathogenic mechanisms in patient subgroups, as considered in this issue by Simon et al. ${ }^{1}$

\section{Eosinophil Biology}

Eosinophils are multifunctional predominantly tissue-dwelling leukocytes with functions in innate immunity, inflammation and other likely more homeostatic host responses. Typically eosinophils in the blood are $<0.5 \times 10^{9} / \mathrm{L}$ (e.g, often $<5 \%$ of leukocytes), although organ infiltration of eosinophils in some diseases (e.g., eosinophilic pneumonias) may not be accompanied by enhanced blood eosinophilia. Eosinophil precursors differentiate in the bone marrow (BM) mediated by specific transcription factors, and eosinophil maturation occurs in response to eosinophilopoietic cytokines (interleukin (IL)-3, granulocyte-macrophage colony stimulating factor, and most importantly IL-5).2 IL-5 also promotes egress of matured eosinophils from the marrow. Eosinophils transit through the blood before migrating into tissues. Specific selectins, adhesion molecules, chemokines (e.g., eotaxins), and cytokines (IL-5) successively and collectively contribute to eosinophil homing.3 In the healthy subject, eosinophils are located in the spleen, lymph nodes, digestive tract (with the exception of the esophagus), thymus, mammary glands, and uterus, where their physiological functions remain largely unknown.

Eosinophils are complex cells that contain and are able to elaborate an array of molecules with very diverse functions. ${ }^{3,4}$ Eosinophil granules contain four highly cationic proteins, major basic protein, eosinophil cationic protein, eosinophil peroxidase, and eosinophil-derived neurotoxin, that upon release exhibit varied cytotoxic and other activities. Reactive oxygen species represent another means of causing damage in the nearby environment. Eosinophils produce lipids, including leukotriene $\mathrm{C}_{4}$, platelet activating factor and prostaglandins, that mediate effects on bronchial and vascular smooth muscle tone, vascular permeability, and chemotaxis. Eosinophils also contain diverse cytokines and chemokines preformed within their granules and secretory vesicles enabling communication with other cells, resulting in enhancement, regulation, and/or repair of inflammation. Eosinophils may secrete their products selectively and differentially, in response to specific ligands, but the exuberant release of granule-derived proteins, by degranulation or cytolysis, may contribute to the tissue damage associated with eosinophil-associated diseases.

\section{Hypereosinophilia}

A number of clinical conditions can lead to enhanced eosinophilopoiesis within the marrow and consequent increased levels of blood eosinophils, termed "eosinophilia", which is defined as blood eosinophil counts exceeding $0.5 \times 10^{9} / \mathrm{L}$. Three levels of severity of eosinophilia have been defined as follows: mild 0.5-1.5 $\times 10^{9} / \mathrm{L}$, moderate $01.5-5 \times 10^{9} / \mathrm{L}$, and severe $>5 \times 10^{9} /$ L. The term "hypereosinophilia", as used in this Perspective article, refers to eosinophil levels above $1.5 \times 10^{9} / \mathrm{L}$; i.e. hypereosinophilia which may intrinsically cause tissue and organ damage, regardless of underlying etiology. In many cases, polyclonal eosinophil expansion occurs in response to over-production of IL-5; the resulting eosinophilia is said to be "reactive" or "secondary". Sources of IL-5 produced in sufficient quantities to stimulate eosinophilopoiesis are relatively limited and include activated T-cells with a "type-2" cytokine profile (most often $\mathrm{CD}^{+}$helper T-cells (Th2 cells), which differentiate in response to parasitic helminth infections or allergens), as well as malignant cells in the setting of solid tumors (mainly adenocarcinomas) or lymphomas (namely Hodgkin's disease and T-cell lymphoma). Rarely, the eosinophilic expansion is clonal, resulting from a hematopoietic stem cell mutation; eosinophils can either represent the major cell-type affected by the cytogenetic abnormality (e.g., in acute or chronic eosinophilic leukemia) or be one of several involved cell-types (e.g., chronic myelomonocytic leukemia, or systemic mast cell disease). 
While eosinophilia can be assessed based on numbers of eosinophils in the blood, of greater concern clinically is the potential for eosinophils recruited into varied tissues to cause damage and dysfunction. The eosinophil blood count is determined both by the level of eosinophil production in the marrow, and the rate of migration of eosinophils from the bloodstream into tissues in response to local secretion of potent chemotactic agents. Levels of blood eosinophilia are an imperfect correlate of the potential for eosinophil-mediated tissue damage. On the one hand, blood eosinophil numbers may be normal in the face of significant eosinophil recruitment into organs, as found in acute and chronic eosinophilic pneumonias. On the other hand, eosinophilia may be present without evidence of tissue damage. The somewhat arbitrary threshold of hypereosinophilia of $1.5 \times 10^{9} / \mathrm{L}$ is classically considered as the level above which organ damage is more likely to occur, but there is no reliable level of blood eosinophilia that precisely reflects the capacity of eosinophils recruited and activated within tissues to cause organ damage. It is within tissues that activated eosinophils degranulate, releasing their preformed mediators, and produce a number of molecules de novo, leading to local damage and dysfunction. Depending on the target organs, various clinical manifestations/complications, including thrombotic complications, can occur. Most tissues and organs can potentially be affected by eosinophilic inflammation, including the skin, lungs, gastrointestinal tract, heart, and central and peripheral nervous systems.

\section{Disorders associated with "secondary" eosinophilia}

An initial consideration of etiologies for eosinophilia needs to focus on several not uncommon causes. Helminthic parasites typically elicit IL-5-mediated eosinophil expansion. Eosinophilia can be constant or fluctuate over time, and any level of severity may be observed. Histories of geographic exposures during travels, immigration or foreign service (even decades before) and recent dietary histories (e.g, trichinellosis) are germane. Toxocariasis can be contracted in any country, following ingestion of soil/vegetables contaminated by excrements of infected dogs, and is often relatively asymptomatic; serological testing is therefore recommended in all eosinophilic patients. Of greatest concern is underlying infection with Strongyloides stercoralis, whose infective larvae penetrate the skin through contact with soil or water contaminated with human feces. This is the only major helminthic parasite with the capacity to propagate itself internally for decades after initial infection, to cause variable eosinophilia (with or without other symptoms) and to result in unleashed disseminated infections in recipients of glucocorticosteroids. Strongyloides is endemic in tropical and subtropical climates and may be contracted sporadically in temperate regions, including the southern states of the US, especially in rural and poor socio-economical habitats (inhabited by carriers who have emigrated from endemic regions). While stool exams for ova and parasites are warranted in patients with eosinophilia, these exams are insensitive for Strongyloides. Therefore patients with a travel history and/or habitat compatible with acquisition of Strongyloides infection should be screened for serum antibodies by ELISA assays. Other helminths lack the capacity for exacerbation of disease with steroids and should be considered principally based on geographic exposure histories.

Allergic and immunologically-mediated diseases to be considered include more common allergic diseases, although eosinophilia $>1.5 \times 10^{9} / \mathrm{L}$ is uncommon in most with asthma. ${ }^{5}$ More pronounced eosinophilia in association with asthma should prompt a consideration of the Churg-Strauss syndrome or allergic bronchopulmonary aspergillosis.

Adverse reactions to medications, both herbal and prescribed, must be considered. While it is assumed that eosinophilia associated with adverse drug reactions is IL-5 mediated, in many instances the mechanism and the organ-targeted involvements elicited by medications remain unclear. Drug-induced eosinophilia may develop without other manifestations of adverse drug reactions, such as rashes or drug fevers, but should nevertheless prompt an evaluation of 
whether organs, including the lungs, kidneys, and heart, are involved. Identifying the culprit agent in patients taking multiple drugs may be challenging. Clinicians may be guided by the type of medications (some being more likely responsible for an eosinophilic drug reaction than others; e.g. anticonvulsants, semi-synthetic penicillins, allopurinol), and by specific targetorgan involvement (hepatitis or the DRESS syndrome (drug-induced rash, eosinophilia, and systemic symptoms)) with anti-convulsants, pneumonitis with nitrofurantoin, semi-synthetic penicillins, and non-steroid anti-inflammatory agents, nephritis with cephalosporins, hypersensitivity vasculitis with allopurinol and phenytoine). In patients with drug-induced pulmonary eosinophilia, blood eosinophilia is usually, but not always, present; if absent, sputum or bronchoalveolar lavage eosinophilia is necessary to help make the diagnosis. In drug-induced acute interstitial nephritis, eosinophilia is common in the involved kidneys, urine, and at times, the blood. Acute necrotizing eosinophilic myocarditis is a serious but uncommon type of hypersensitivity myocarditis, with reactions to medications, responsible in some cases. A syndrome of hepatitis with eosinophilia can be a manifestation of drug reactions. Other medication-related eosinophilic responses include drug-induced hypersensitivity vasculitis, the DRESS syndrome, and forms of gastroenterocolitis.

Besides these relatively common causes of eosinophilia, neoplastic diseases, including varied adenocarcinomas, some forms of Hodgkin's disease, T cell lymphoma, and mastocytosis, may be associated with paraneoplastic eosinophilia, as noted above.

Other infrequent causes include rare immunodeficiency disorders, namely hyper-IgE syndrome and Omenn's syndrome. Organ-specific eosinophilic disorders may also be associated with blood eosinophilia; these include acute and chronic eosinophilic pneumonias, eosinophilic gastroenteritides, and some principally skin diseases.

\section{Hypereosinophilic syndromes}

When an underlying etiology for persistent hypereosinophilia is not identified despite thorough diagnostic evaluation, clinicians must consider the diagnosis of hypereosinophilic syndromes (HES), which comprise a heterogeneous group of uncommon disorders. The original defining criteria for HES, proposed in $1975,{ }^{6}$ include: 1 ) blood eosinophilia $\geq 1.5 \times 10^{9} / \mathrm{L}$ lasting for $>$ 6 months, 2) no evidence for an underlying condition known to cause hypereosinophilia, and 3 ) existence of eosinophil-mediated organ damage and/or dysfunction. These criteria no longer reflect clinical practice which integrates modern diagnostic and management facilities and recent advances in pathogenic mechanisms.7 In this issue, Simon et al summarize the shortcomings of the original criteria and propose a series of contemporary diagnostic modifications. ${ }^{1}$ Briefly, "persistent" hypereosinophilia is no longer defined by a 6-month duration, but can be shorter provided other causes of hypereosinophilia have been excluded. Several eosinophil-associated diseases, previously considered as distinct from HES, have been integrated in recent HES classification schemes; these include the Churg-Strauss syndrome, and organ-specific eosinophilic disorders such as chronic eosinophilic pneumonia and eosinophilic gastrointestinal disorders (discussed elsewhere in this issue). Target-organ damage need not be present at the outset to satisfy the diagnosis of HES.

Clinically, HES include potentially lethal multi-system disorders, characterized by eosinophilic infiltration of a variable spectrum of target organs, predominantly the skin, heart, lungs, gastrointestinal tract, and central and peripheral nervous systems. ${ }^{7}$ Involvement of other organs, hepato- and/or splenomegaly, and microvascular thrombotic phenomena related to endothelial damage, occur with variable frequencies. The nature and severity of organ damage are extremely variable from one patient to another and often unpredictable. For a given level of blood eosinophilia, some patients develop relatively mild disease presentations, such as isolated cutaneous manifestations that may not warrant therapy, whereas at the other end of 
the spectrum, some present with rapidly progressive heart failure or thrombotic complications that require urgent medical attention. In some forms of HES, disease evolution in a minority of patients may be complicated by the development of malignancies involving either myeloid (acute myelogenous or eosinophilic leukemia) or lymphoid cells (peripheral T-cell lymphoma).

Recent studies have led to the identification of two major pathogenically identifiable variants of HES. Myeloproliferative variant HES (M-HES) is characterized by features that are typically encountered in other myeloproliferative diseases, including increased serum vitamin B12, hepatomegaly, splenomegaly, anemia, thrombocytopenia, circulating myeloid precursors, and increased BM cellularity with a left-shift in maturation. The majority of patients with M-HES have a cryptic interstitial deletion on chromosome 4q12 that results in expression of a FIP1L1PDGFRA (FIP1-like 1/ Platelet derived growth factor receptor alpha) fusion protein with autonomous tyrosine kinase activity ("F/P-associated" HES or chronic eosinophilic leukemia). 8 Although this appears to be a stem-cell mutation, clonal eosinophil expansion dominates over other lineages. Clinical characteristics of this variant include strong male predominance, increased serum vitamin B12 and tryptase levels, mucosal ulcers, splenomegaly, endomyocardial fibrosis as well as other organ-based fibrotic complications, and possible progression towards acute eosinophilic leukemia or blast crisis.9 Much less commonly translocations on chromosomes 5q33 and 8p11 lead, respectively, to platelet-derived growth factor receptor beta (PDGFRB)- and fibroblast growth factor receptor 1 (FGFR1) -rearranged clonal forms of M-HES.10

The lymphocytic HES (L-HES) variant is characterized by polyclonal eosinophil expansion in response to marked over-production of IL-5 by deregulated T-cells in vivo. ${ }^{11}$ These T-cells can be detected on the basis of abnormal surface phenotypes, including $\mathrm{CD}^{-} \mathrm{CD}^{+}$and $\mathrm{CD}^{+} \mathrm{CD}^{-} \mathrm{CD}^{-}$, and are sometimes monoclonal. Published case reports indicate that these $\mathrm{T}$ cell clones may be prone to malignant transformation (T-cell lymphoma). Clinically, patients often present with predominant cutaneous manifestations, although other organs may be targeted as well, increased serum IgE levels and hypergammaglobulinemia.

Together, F/P-associated M-HES and L-HES represent only about a quarter to a third of those with HES. ${ }^{12}$ In the majority of patients with HES, the etiology remains to be delineated. For those with myeloid disorders and eosinophilia, the World Health Organization has also presented an updated 2008 classification scheme. ${ }^{13}$

\section{Practical approach to the hypereosinophilic patient}

The clinician confronted with a patient presenting with hypereosinophilia (Fig 1) must first and foremost address two questions: 1) is hypereosinophilia secondary to a common and treatable underlying condition such as parasitic infections or adverse drug reactions? and 2) is hypereosinophilia in itself causing rapidly progressive damage?

Given the diverse nature of therapeutic compounds that have been reported to trigger eosinophilic allergic reactions, any agent the patient may be taking (including herbal compounds and nutritional supplements) is a potential suspect. Careful history taking should try to elucidate the sequence of events from introduction of a new treatment to discovery of hypereosinophilia and/or appearance of symptoms. However, some reactions may develop months and even years after initiation of therapy. The clinician should therefore withdraw any agent that is not crucial for the patient's well-being. In the absence of organ involvement, eosinophilia by itself need not mandate cessation of drug therapy, if such is medically indicated. If organ involvement and/or systemic symptoms develop, cessation of drug administration is warranted. In acute reactions, identification of the offending agent is often straight-forward; the chronology of drug initiation as well as the type of molecule itself (e.g. anti-convulsants, 
anti-microbials) facilitate the process. In other cases, it may prove to be more challenging, and the clinician must proceed by trial-and-error.

Parasitic infections, as noted above, represent another common cause of hypereosinophilia that must be investigated in all cases. The patient's travel history and habitat will dictate the type of parasites to look for using serological tests and microscopic examination of stools, urine, and/or blood. Although a comprehensive approach to diagnosis of helminth-elicited eosinophilia is not within the scope of this perspective article, the clinician should be aware of the potential gravity of infection with $S$. stercoralis, as noted above. The capacity of this parasite to cause disseminated, hyperinfection syndrome in patients treated with corticosteroids mandates that serological testing for this parasite should be performed in hypereosinophilic patients who have traveled to an endemic area at any time in their life or who live in temperate regions in close community with emigrants from endemic areas.

Practically, the initial work-up of a hypereosinophilic patient therefore mandates careful examination of drug history, withdrawal of non-essential agents and those which are particularly suspect (such as anti-convulsants, anti-microbials), serological testing for selected helminths, examination of three stools for ova and larvae, and appropriate anti-parasitic treatment if these tests are positive. If hypereosinophilia persists despite these measures (or if these conditions are not found), other causes of secondary hypereosinophilia must be investigated. Serological, imaging, endoscopic, and histological studies should be selected on the basis of the most likely causes in light of risk factors, signs and symptoms, patient history and physical examination. The finding of an atopic background or other conditions known to be associated with low-grade hypereosinophilia, such as hypoadrenalism or psoriasis, should not be considered a sufficient explanation for eosinophil levels $\geq 1.5 \times 10^{9} / \mathrm{L}$. Special attention should be paid to the possibility of an underlying malignant disorder causing paraneoplastic hypereosinophilia. Although investigations will vary from one patient to another, the following are recommended in all cases: complete blood count and differential, peripheral blood smear looking for dysplastic eosinophils or blasts, serum tryptase, serum vitamin B12, IgE, and cardiac troponin levels, ANCA (anti-neutrophil cytoplasmic antibody), electrocardiogram (EKG), echocardiogram, pulmonary function tests (PFT), and a thoraco-abdominal computed tomography (CT)-scan.

These studies can be valuable for detecting and assessing severity of eosinophil-mediated organ damage. Whether eosinophil expansion is secondary to an identifiable disease or not, the potential complications related to tissue and organ infiltration are identical. The most serious complications of hypereosinophilia that require urgent eosinophil-lowering measures, are myocardial damage, pulmonary involvement with hypoxia, and neurological involvement. Splinter hemorrhages, likely reflecting endocardial formation of thrombi, and elevated serum troponin levels are indicative of cardiac involvement. Should one of these findings be detected at initial evaluation, high-dose corticosteroid treatment should be initiated promptly ( $1 \mathrm{mg} / \mathrm{kg}$ daily, followed by $15 \mathrm{mg} / \mathrm{kg}$ IV on three consecutive days in the absence of an initial response). If there is any possibility that the patient may have contracted Strongyloides infection even in the distant past (i.e. travel to or prior residence in any country where this parasite is endemic), ivermectin $(200 \mu \mathrm{g} / \mathrm{kg}$ on two consecutive days) should be given concomitantly, even if results of serology are not yet available, because of the risk that corticosteroids could unleash a hyperinfection syndrome. Other acute eosinophil-lowering agents should be reserved for corticosteroid non-responders and preferably be prescribed by specialists; these include vincristine (1-2 gr IV) and in selected cases imatinib mesylate (400 mg/day).

Situations wherein urgent eosinophil-lowering therapy is typically required in addition to specific therapeutic measures include DRESS (drug-induced rash with eosinophilia and systemic symptoms) for which withdrawal of the offending agent does not result in immediate 
resolution of hypereosinophilia, and paraneoplastic hypereosinophilia complicated by eosinophil-mediated heart failure.

Failure to detect an underlying cause of eosinophilia should prompt initiation of a further diagnostic tests aiming to identify one of the HES variants. If initial clinical assessments have shown that eosinophil-lowering therapy should be given without delay, blood and BM samples should be obtained for these tests beforehand whenever possible. The following are currently recommended for all patients fulfilling HES diagnostic criteria (see Simon et al) ${ }^{1}$ : search for presence of the F/P fusion using both RT-PCR and FISH (CHIC2 deletion), T cell receptor (TCR) phenotyping, PCR analysis of TCR gene rearrangement patterns, and chromosomal karyotyping. The initial studies evaluating causes and complications of hypereosinophilia should be informative as to the presence of clinical and laboratory features often associated with M-HES and L-HES. Identification of patients with these two HES variants has implications in terms of treatment and follow-up. Patients with F/P-associated HES respond extremely well to imatinib, that should be initiated as first-line therapy without delay, at a starting dose of 100-400 mg daily, depending on the severity of complications. All patients, even asymptomatic, should be treated; the goal of therapy is cytogenetic and molecular remission. Such patients often are unresponsive to corticosteroid therapy. However, if there is evidence of cardiac involvement at diagnosis, corticosteroids should be given concomitantly with initiation of imatinib to prevent acute myocarditis. For patients with L-HES, a moleculartargeted approach to therapy has yet to be defined, and corticosteroids remain first-line treatment. Knowledge that T-cells are the primarily affected lineage in L-HES should direct the choice of second-line and/or corticosteroid-sparing agents, favoring molecules which affect T-cell functions (e.g. interferon (IFN)- $\alpha$ and perhaps alemtuzumab in selected cases). Regular assessment of patients with L-HES is warranted for the detection of T-cell lymphoma, using thoraco-abdominal CT-scans and fluoro-deoxy-glucose positron emission tomography (FDGPET).

In the majority of cases of HES, not due to M-HES or L-HES variants, depending on the nature and severity of clinical complications of hypereosinophilia, treatment of idiopathic HES may or may not be necessary, depending on evidence of associated organ involvement.

Corticosteroids represent an effective first-line approach to lowering eosinophils in the majority of cases. ${ }^{12}$ However, the dose required to stabilize disease and drug tolerance are variable from one patient to another. If the required maintenance dose is $>10 \mathrm{mg} / \mathrm{day}$, and/or in presence of significant side effects, introduction of a corticosteroid-sparing agent is recommended in order to reduce short- and long-term corticosteroid-induced toxicity. Hydroxyurea and INF- $\alpha$ are the most frequently prescribed second-line agents, and a short course of imatinib may be worthwhile in patients with features of myeloproliferative disease. These agents are also used for corticosteroid non-responders. Mepolizumab, an anti-IL-5 antibody, has recently been shown to be an extremely well-tolerated and effective corticosteroid-sparing agent in patients with F/P-negative HES; this promising agent may or may not be currently available in the setting of compassionate use programs.

Details on how therapy and follow-up should be conducted in patients with the known HES variants and idiopathic HES can be found in several comprehensive recent review articles. ${ }^{7,10}$

In conclusion, hypereosinophilia is potentially harmful regardless of the nature of the underlying condition and requires careful management. Appropriate treatment is dependent in part on the ability to make an accurate etiologic diagnosis, and to judge the clinical urgency for introducing an eosinophil-lowering agent. Advances in understanding the pathogenesis of HES variants have resulted in improved outcome for these uncommon causes of persistent hypereosinophilia. 


\section{Abbreviations}

$\begin{array}{ll}\text { BM } & \text { Bone marrow } \\ \text { CBC } & \text { complete blood cell count } \\ \text { CS } & \text { corticosteroid } \\ \text { CT } & \text { computed tomography } \\ \text { DRESS } & \text { syndrome } \\ \text { drug-induced rash } & \text { eosinophilia, and systemic symptoms syndrome } \\ \text { EKG } & \text { electrocardiogram } \\ \text { FDG-PET } & \text { fluoro-deoxy-glucose positon emission tomography } \\ \text { FISH } & \text { fluorescent in situ hybridization } \\ \text { F/P } & \text { FIP1-like 1/ Platelet derived growth factor receptor alpha fusion } \\ \text { HES } & \text { hypereosinophilic syndromes } \\ \text { IL } & \text { interleukin } \\ \text { PDGFRA } & \text { platelet-derived growth factor receptor alpha } \\ \text { PFT } & \text { pulmonary function test } \\ \text { RTPCR } & \text { reverse transcription polymerase chain reaction } \\ \text { TCR } & \text { T cell receptor }\end{array}$

\section{References}

1. Simon H-U, et al. Re-definition of hypereosinophilic syndrome. J Allergy Clin Immunol. 2010 this issue.

2. Ackerman SJ, Bochner BS. Mechanisms of eosinophilia in the pathogenesis of hypereosinophilic disorders. Immunol Allergy Clin North Am 2007;27:357-75. [PubMed: 17868854]

3. Blanchard C, Rothenberg ME. Biology of the eosinophil. Advances in immunology 2009;101:81-121. [PubMed: 19231593]

4. Spencer LA, Szela CT, Perez SA, Kirchhoffer CL, Neves JS, Radke AL, Weller PF. Human eosinophils constitutively express multiple Th1, Th2, and immunoregulatory cytokines that are secreted rapidly and differentially. J Leukoc Biol 2009;85:117-23. [PubMed: 18840671]

5. Bousquet J, Chanez P, Lacoste JY, Barneon G, Ghavanian N, Enander I, Venge P, Ahlstedt S, SimonyLafontaine J, Godard P, Michel FB. Eosinophilic inflammation in asthma. N Engl J Med 1990;323:1033-1039. [PubMed: 2215562]

6. Chusid MJ, Dale DC, West BC, Wolff SM. The hypereosinophilic syndrome: analysis of fourteen cases with review of the literature. Medicine (Baltimore) 1975;54:1-27. [PubMed: 1090795]

7. Klion A. Hypereosinophilic syndrome: current approach to diagnosis and treatment. Annu Rev Med 2009;60:293-306. [PubMed: 19630574]

8. Cools J, DeAngelo DJ, Gotlib J, Stover EH, Legare RD, Cortes J, et al. A tyrosine kinase created by fusion of the PDGFRA and FIP1L1 genes as a therapeutic target of imatinib in idiopathic hypereosinophilic syndrome. N Engl J Med 2003;348:1201-14. [PubMed: 12660384]

9. Bain BJ, Fletcher SH. Chronic eosinophilic leukemias and the myeloproliferative variant of the hypereosinophilic syndrome. Immunol Allergy Clin North Am 2007;27:377-88. [PubMed: 17868855]

10. Tefferi A, Gotlib J, Pardanni A. Hypereosinophilic syndrome and clonal eosinophilia: point-of-care diagnostic algorithm and treatment update. Mayo Clin Proc 2010;85:158-164. [PubMed: 20053713]

11. Roufosse F, Cogan E, Goldman M. Lymphocytic variant hypereosinophilic syndromes. Immunol Allergy Clin North Am 2007;27:389-413. [PubMed: 17868856] 
12. Ogbogu PU, Bochner BS, Butterfield JH, Gleich GJ, Huss-Marp J, Kahn JE, et al. Hypereosinophilic syndrome: A multicenter, retrospective analysis of clinical characteristics and response to therapy. $\mathrm{J}$ Allergy Clin Immunol 2009;124:1319-1325. [PubMed: 19910029]

13. Vardiman JW, Thiele J, Arber DA, Brunning RD, Borowitz MJ, Porwit A, et al. The 2008 revision of the World Health Organization (WHO) classification of myeloid neoplasms and acute leukemia: rationale and important changes. Blood 2009;114:937-951. [PubMed: 19357394] 


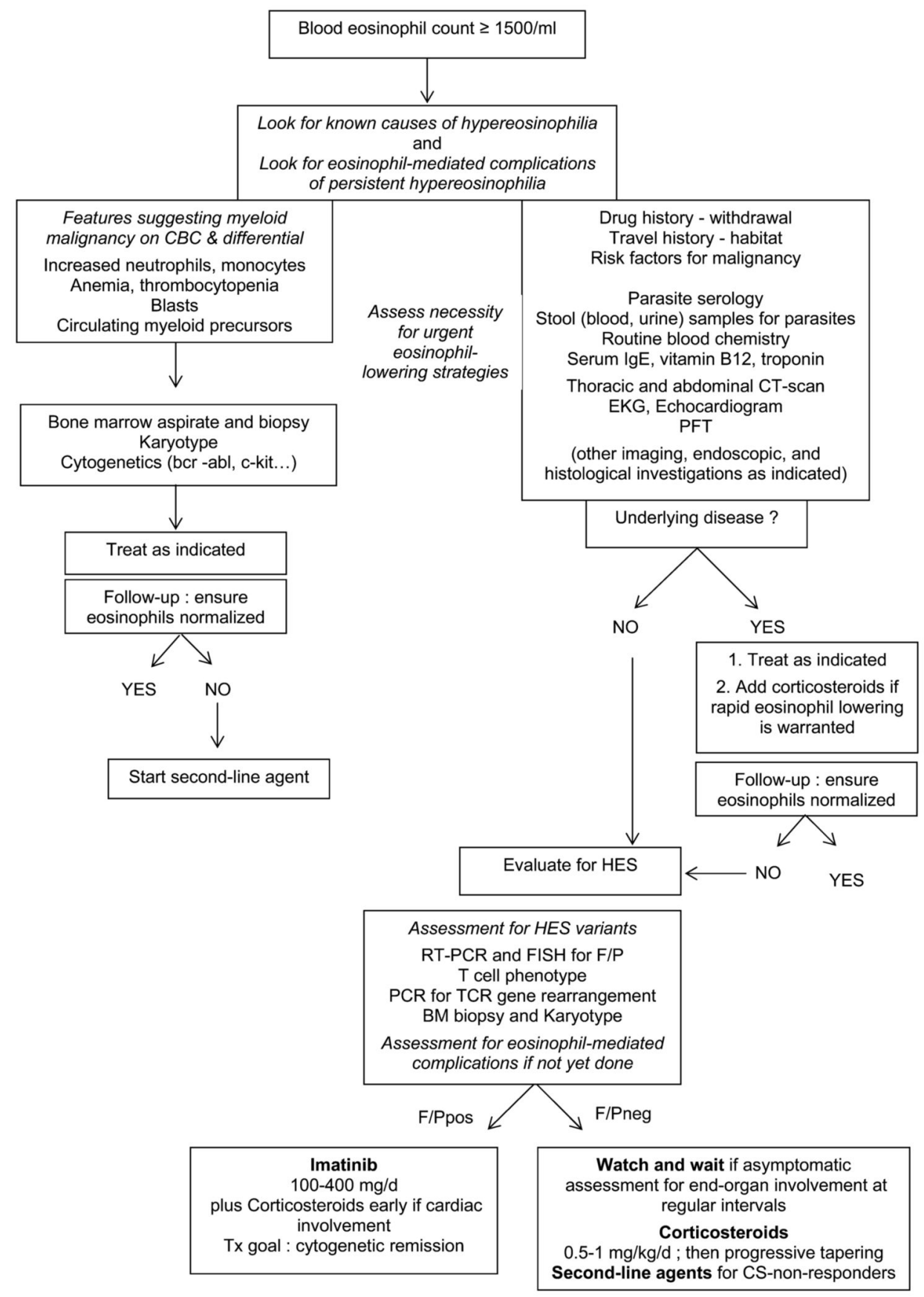

Figure 1.

Approach to the diagnosis and treatment of patients with hypereosinophilia. 